Homology, Homotopy and Applications, vol.5(1), 2003, pp.251-260

\title{
ON SIGNATURES AND A SUBGROUP OF A CENTRAL EXTENSION TO THE MAPPING CLASS GROUP
}

\author{
JONATHAN NATOV \\ (communicated by Jonathan Rosenberg)
}

\begin{abstract}
Atiyah's work [1] describes the relationship between multiplication in a central extension of the mapping class group of a surface of genus $n$ and the signatures of 4-dimensional manifolds. This work studies a subgroup of the central extension, which comes from the image of a representation of the pure framed braid group on $n$-strands found in [5], and the signatures of corresponding 4-manifolds via a split exact sequence. We construct a splitting map to prove the sequence is split exact, and we use the splitting to give a topological description of homology classes in 4-dimensional manifolds with non-zero intersection. We conclude with a description of multiplication in the subgroup.
\end{abstract}

\section{Introduction}

In his paper [1], M. Atiyah uses 2-framings to place Witten's 3-manifold invariant in the context of algebraic topology. Witten's invariant relies on 2-framings, which are related to a central extension $\overline{\Gamma_{n}}$ of the mapping class group, $\Gamma_{n}$, of a compact oriented surface of genus $n$. In [1] Atiyah proves the sequence

$$
0 \rightarrow \mathbb{Z} \rightarrow \overline{\Gamma_{n}} \rightarrow \Gamma \rightarrow 0
$$

is an exact sequence. $\overline{\Gamma_{n}}$ consists of pairs $(f, \alpha)$ with $f \in \Gamma_{n}$, and $\alpha$ a 2 -framing on a 3-manifold.

Given an oriented compact 3-manifold $Y$ with tangent bundle $T_{Y}$, Atiyah defined a 2-framing as a homotopy class of trivializations of $T_{Y} \oplus T_{Y}$ viewed as a $\operatorname{Spin}(6)$ bundle of the diagonal embedding $S O(3) \rightarrow S O(3) \times S O(3) \rightarrow S O(6)$. He then showed the correspondence between a 2 -framing $\alpha$ and an integer via

$$
\sigma(\alpha)=\operatorname{Sign}(Z)-\frac{1}{6} p_{1}\left(T_{Y} \oplus T_{Y}\right),
$$

where $Z$ is a 4-manifold with boundary $Y$, and $p_{1}$ is the relative Pontrjagin class $p_{1} \in H^{4}(Y \times I, Y \times \partial I)$. Atiyah proved $\sigma$ does not depend on the choice of $Z$.

supported by PSC-CUNY grant 669430

Received October 16, 2001, revised July 16, 2003; published on July 23, 2003.

2000 Mathematics Subject Classification: 57N05, 57M25, 20F36, 57N13.

Key words and phrases: Mapping Class Group, Pure Braids, Signatures.

(C) 2003, Jonathan Natov. Permission to copy for private use granted. 
In this paper we use a representation $\Phi$, see section 2 , from the pure framed braid group $\mathbb{Z}^{n} \oplus P_{n}$ to the mapping class group $\Gamma_{n}$, and study the pullback of the image subgroup in $\overline{\Gamma_{n}}$, which we denote $\overline{\mathbb{Z}^{n} \oplus P_{n}}$.

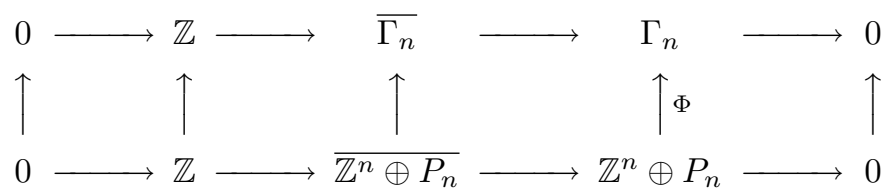

The 4-manifolds in [1] are constructed using mapping tori $X_{f}$ and $X_{g}$, where $X$ is a surface (see definition 3). To explain restricting to $\overline{\mathbb{Z}^{n} \oplus P_{n}}$, in [5] it was shown that any compact orientable 3-manifold can be represented as $X_{f}$, where $f \in i m(\Phi)$ and $X$ is compact oriented surface of genus $n$. Furthermore, the multiplication in $\overline{\mathbb{Z}^{n} \oplus P_{n}}$ is computed via linking matrices coming from the closures of pure framed braids, see theorem 2 of section 5 .

We use the above correspondence between 2-framings and integers to consider elements of $\overline{\mathbb{Z}^{n} \oplus P_{n}}$ to be pairs $(h, c)$, consisting of mapping class elements $h$, and integers $c$. The group multiplication is given by $(f, a) \cdot(g, b)=(f g, a+b+3$. $\left.\operatorname{Sign}\left(W_{f, g}^{4}\right)\right)$, where $\operatorname{Sign}\left(W_{f, g}^{4}\right)$ is the signature of the intersection form

$$
H_{2}\left(W_{f, g}^{4}, \mathbb{Z}\right) \times H_{2}\left(W_{f, g}^{4}, \mathbb{Z}\right) \rightarrow \mathbb{Z} .
$$

$H_{2}\left(W_{f, g}^{4}\right)$ is computed using a Mayer Vietoris sequence, see equation 1 of section 3. We construct a splitting map $\tau$ of section 4 to show the Mayer Vietoris sequence is split exact. In section 4 we use $\tau$ to construct specific examples of classes in $H_{2}\left(W_{f, g}^{4}\right)$ with non zero intersection. Note: we compute homology groups over the integers.

\section{The representation $\Phi: \mathbb{Z}^{n} \oplus P_{n} \rightarrow \Gamma_{n}$}

Let $B_{n}, n>1$, be the group given by generators $\sigma_{1}, \ldots, \sigma_{n-1}$ and relations

1) $\sigma_{i} \sigma_{j}=\sigma_{j} \sigma_{i}$ for $|i-j| \geqslant 2$

2) $\sigma_{i} \sigma_{i+1} \sigma_{i}=\sigma_{i+1} \sigma_{i} \sigma_{i+1}$

$B_{n}$ is called the Braid Group on $n$-strands. Let $\Sigma_{n}$ denote the symmetric group acting on $\{1,2, \cdots, n\}$, and $\pi: B_{n} \rightarrow \Sigma_{n}$ denote the homomorphism sending $\sigma_{i}$ to the transposition $(i, i+1)$. Let $P_{n}$ denote the kernel of $\pi$.

Definition 1. The pure framed braid group on n-stands is the direct sum $\mathbb{Z}^{n} \oplus P_{n}$, where $\mathbb{Z}^{n}$ denotes the group of $n$-tuples of integers under addition. 


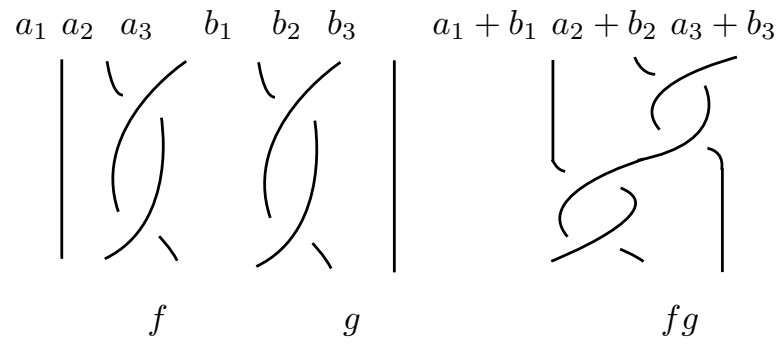

Figure 1: Multiplication of pure framed braids.

We will use a geometric description of pure framed braids. The equivalence between the algebraic and geometric definitions is found in [2]. Consider a cylinder $D^{2} \times[0,1]$ with projection $\pi_{1}: D^{2} \times[0,1] \rightarrow D^{2}$ defined $\pi_{1}(x, t)=x$. Choose $n$ non-intersecting (smooth) curves in $D^{2} \times[0,1]$, where each curve intersects $D^{2} \times\{t\}$ in exactly one point, for $0 \leqslant t \leqslant 1$. If $x_{i}$ and $y_{i}$ denote the points the $i^{t h}$ curve intersects $D^{2} \times\{0\}$ and $D^{2} \times\{1\}$ respectively, then $\pi_{1}\left(x_{i}\right)=\pi_{1}\left(y_{i}\right)$ for $1 \leqslant i \leqslant n$. We require the $x_{i}$ to be evenly spaced along a fixed diameter in the interior of $D^{2} \times\{0\}$. In Figure 1 we see the projection of pure framed braids on 2 strands into the plane. We use breaks in the curves to indicate one strand passing over another. The framed pure braid has an integer assigned to each curve called the framing number. Two pure framed braids are equivalent if they are isotopic via an isotopy of $D^{2} \times[0,1]$ which fixes the end-points of the curves and preserves the framings. We illustrate the multiplication in figure 1 with $\left(a_{1}, a_{2}, a_{3}\right) f$ and $\left(b_{1}, b_{2}, b_{3}\right) g \in Z^{3} \oplus P_{3}$. Their product $\left(a_{1}+b_{1}, a_{2}+b_{2}, a_{3}+b_{3}\right) f g$ is formed by stacking $f$ on top of $g$ and adding the corresponding framings. Note: we omit a framing number if it is zero.

Notation 1. By $D_{n}$ we mean a (unit) disc $D^{2}$ with $n$ open disjoint discs removed from the interior. The removed discs were chosen so that their centers were evenly spaced on a fixed diameter of $D^{2}$.

Let $p f b\left(D_{n}\right)$ be the group of isotopy classes of homeomorphisms from $D_{n}$ to $D_{n}$ which fix the $\partial D_{n}$ pointwise.

Theorem 1. (Prasolov, V. V. and Sossinsky, A. B.) pfb $\left(D_{n}\right)$ is isomorphic to $\mathbb{Z}^{n} \oplus P_{n}$.

The proof appears in [6]. To give a description of the isomorphism, let $[g] \epsilon$ $p f b\left(D_{n}\right)$, and $g_{0}$ be a representative. To obtain a pure framed braid corresponding to $[g]$, we extend the map $g_{0}$ to $g: D^{2} \rightarrow D^{2}$ by $g(x)=x$ for $x$ not in the interior of $D_{n}$. This extension is possible because $g_{0}(x)=x$ for $x \in \partial D_{n}$. Now define an isotopy $I: D^{2} \times[0,1] \rightarrow D^{2}$,

$$
I(x, t)= \begin{cases}x, & \text { if } t=0 \\ x, & \text { if }|x|>t \\ t g\left(t^{-1} x\right), & \text { otherwise }\end{cases}
$$

Note $I(x, 0)=x$ and $I(x, 1)=g(x)$. In the complement of the interior of $D_{n}$ in $D^{2}$ there are $n$ closed discs. If $c_{i}$ is the center of the $i^{t h}$ disc, then $\left(I\left(c_{i}, t\right), t\right)$ for 
$0 \leqslant t \leqslant 1$ is an arc in $D^{2} \times[0,1]$. The $n$ arcs are the $n$ strands of the pure braid. Choose any point $b_{i}$ on the $S^{1}$ boundary of the disc with center $c_{i}$. The linking number of $\left(I\left(b_{i}, t\right), t\right)$ with $c_{i} \times[0,1]$ is an integer, and this will be the framing corresponding to the $i^{\text {th }}$ strand. For example, in figure 2 we have a Dehn twist about the dashed curve, which is an element of $p f b\left(D_{3}\right)$. The pure framed braid is formed by following the image of the interior points $c_{1}, c_{2}, c_{3}$ under the isotopy $I$.

Definition 2. The mapping class group of a (compact oriented) genus $n$ surface, denoted here by $\Gamma_{n}$, is the group of isotopy classes of orientation preserving homeomorphisms from the surface to itself.

An element of $p f b\left(D_{n}\right)$ is an isotopy class of maps of $D_{n} \rightarrow D_{n}$, or equivalently $D_{n} \times\{1\} \rightarrow D_{n} \times\{1\}$. Each representative of the isotopy class, fixes $\partial\left(D_{n} \times\{1\}\right)$ pointwise, and may be extended by the identity map to

$\partial\left(D_{n} \times[0,1]\right)$. This extension is a homomorphism $p f b\left(D_{n}\right) \rightarrow \Gamma_{n}$. Composing this map with the isomorphism $\mathbb{Z}^{n} \oplus P_{n} \rightarrow p f b\left(D_{n}\right)$ yields $\Phi: \mathbb{Z}^{n} \oplus P_{n} \rightarrow \Gamma_{n}$. Our viewpoint for the construction of $\Phi$ was inspired by [3] in which the authors discussed representations of platts.
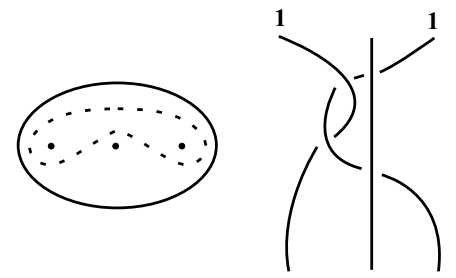

Figure 2: A Dehn twist and corresponding pure framed braid.

\section{The 4-manifold $W_{f, g}^{4}$}

The construction of $W_{f, g}^{4}$ from [1] requires the definition of a mapping torus.

Definition 3. For a homeomorphism $f$ of a surface $X$ to itself, define the mapping torus $X_{f}$ as $\frac{X \times[0,1]}{(p, 0) \sim(f(p), 1)}$

We are interested in the case where $X=\partial\left(D_{n} \times[0,1]\right)$, a surface of genus $n$, and $f$ a representative of $[f]$ in the image of $\Phi: \mathbb{Z}^{n} \oplus P_{n} \rightarrow \Gamma_{n}$. We can view $X_{f}$ as a fiber bundle over $S^{1}$, with fiber $\partial\left(D_{n} \times[0,1]\right)$, and $X_{f} \times[0,1]$ as a fiber bundle over an annulus. The 4 -manifold $W_{f, g}^{4}$ is defined as the quotient space of $\left(X_{f} \times[0,1]\right) \cup\left(X_{g} \times[0,1]\right)$ where, for $t \in[0.5,0.75]$, we identify

$\left(\partial\left(D_{n} \times[0,1]\right) \times\{t\}\right) \times\{1\}$ of $X_{f} \times[0,1]$ with $\left(\partial\left(D_{n} \times[0,1]\right) \times\{t\}\right) \times\{1\}$ of $X_{g} \times[0,1]$ by the identity map $\partial\left(D_{n} \times[0,1]\right) \rightarrow \partial\left(D_{n} \times[0,1]\right)$. Viewing $X_{f} \times[0,1]$ and $X_{g} \times[0,1]$ as fiber bundles over annuli, we have identified arcs on the outer boundary circles of the annuli base spaces and their corresponding fiber. We obtain homeomorphic 4-manifolds if different representatives of $[f]$ and $[g]$ are used. The result is a fiber bundle with base $D_{2}$. Restricting $W_{f, g}^{4}$ to the interior boundary circles of $D_{2}$ we have $X_{f}$ and $X_{g}$. Restricting to the exterior boundary circle of $D_{2}$ we have $X_{f g}$. 
It is shown in [1] that 4 -manifolds $Z$ with $\partial Z=X_{f}+X_{g}-X_{f g}$ have the same signature.
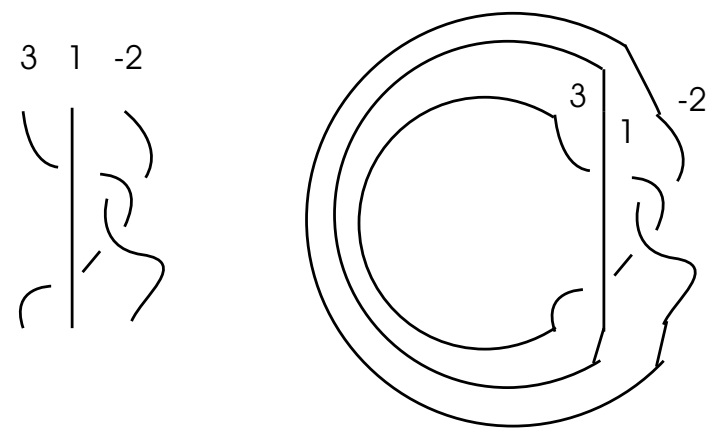

Figure 3: A framed braid and its closure to a framed link.

In order to obtain the signature of $W_{f, g}^{4}$, we compute the homology via a Mayer Vietoris sequence. This sequence is defined with spaces $X_{0}$ and $X_{1}$, whose union is $W_{f, g}^{4}$. The space $X_{1}$ is a fiber bundle over $D_{2}$ with fiber $D_{n}$. It is constructed in the same way as $W_{f, g}^{4}$, except that we replace the surface fiber $\partial\left(D_{n} \times[0,1]\right)$ with $D_{n} \times\{1\}$. The fiber in $X_{1}$ over the two interior boundary circles of the base space $D_{2}$ is homeomorphic to complements of tubular neighborhoods of $\hat{f}$ and of $\hat{g}$ in solid tori, where $\hat{f}$ and $\hat{g}$ denote the closures of the pure braids corresponding to $f$ and $g$. The closure of a framed braid is formed by connecting the two ends of each strand using a simple closed arc. The result is a link with each path component given the framing coming from the corresponding strand, see figure 3 . The space $X_{0}$ is the product space $D_{2} \times\left(D_{n} \times\{0\}\right)$. Gluing two copies of $D_{n}$ along their boundary is homeomorphic to a genus $n$ surface. If at each point in $D_{2}$ we glue the $D_{n} \times\{1\}$ fiber of $X_{1}$ to the $D_{n} \times\{0\}$ fiber of $X_{0}$, the result is $W_{f, g}^{4}$. Using collar neighborhoods of the $D_{n}$ fiber so that $X_{0}$ and $X_{1}$ are open, we obtain the Mayer Vietoris sequence.

$$
\begin{aligned}
\cdots & \longrightarrow H_{2}\left(X_{0}\right) \oplus H_{2}\left(X_{1}\right) \stackrel{\sigma}{\longrightarrow} \quad H_{2}\left(W_{f g}^{4}\right) \\
\stackrel{\delta}{\longrightarrow} H_{1}\left(X_{0} \cap X_{1}\right) & \stackrel{j}{\longrightarrow} H_{1}\left(X_{0}\right) \oplus H_{1}\left(X_{1}\right) \longrightarrow \cdots
\end{aligned}
$$

The map $j: H_{1}\left(X_{0} \cap X_{1}\right) \rightarrow H_{1}\left(X_{0}\right) \oplus H_{1}\left(X_{1}\right)$ takes $a \rightarrow(a, a)$ and $\sigma(b, c)=b-c$. By the exactness of the sequence, $\operatorname{im}(\sigma)=\operatorname{ker}(\delta)$ and $\operatorname{ker}(j)=i m(\delta)$. In Lemma 2 of section 5 we show that the above sequence is split exact. It will then follow that a basis for $H_{2}\left(W_{f g}^{4}\right)$ is isomorphic to a direct sum of a basis for $i m(\sigma)$ with a basis for $\operatorname{ker}(j)$.

\section{Constructing intersecting classes.}

For $[f]$ and $[g]$ in the image of $\Phi: \mathbb{Z}^{n} \oplus P_{n} \rightarrow \Gamma_{n}$ we will define a splitting $\tau: \operatorname{ker}(j) \rightarrow H_{2}\left(W_{f, g}^{4}\right)$. In lemma 3 we show that only elements in the image of $\tau$ can have non-zero intersection. To describe $\operatorname{ker}(j)$ we label curves in $X_{0} \cap X_{1}$, 
which is a fiber bundle with base $D_{2}$ and fiber $\partial D_{n}$; recall $D_{n}$ is a disc $D^{2}$ with interior open discs $\operatorname{int}\left(e_{1}\right), \cdots, \operatorname{int}\left(e_{n}\right)$ removed. Label the boundary circles of $D_{n}$ by $s_{0}, s_{1}, \cdots, s_{n}$, with $s_{0}=\partial D^{2}$. Let $D_{2}$ be the disc $D^{2}$ with the interior of two discs, int $\left(d_{1}\right)$ and $\operatorname{int}\left(d_{2}\right)$, removed.

Notation 2. Let $y_{i}$ be a point of $s_{i}$, and $x \in D_{2}$. Set $p_{i}=\partial d_{1} \times\left\{y_{i}\right\}, q_{i}=$ $\partial d_{2} \times\left\{y_{i}\right\}$ and $m_{i}=s_{i} \times\{x\}$.

Using the Mayer Vietoris sequence in equation (1), along with the Künneth formula for computing the homology of a product space, one obtains homology bases for $H_{1}\left(X_{0} \cap X_{1}\right)$ and $H_{1}\left(X_{0}\right) \oplus H_{1}\left(X_{1}\right)$. Write $j: H_{1}\left(X_{0} \cap X_{1}\right) \rightarrow H_{1}\left(X_{0}\right) \oplus H_{1}\left(X_{1}\right)$ as $j=j_{0} \oplus j_{1}$, so $j_{1}: H_{1}\left(X_{0} \cap X_{1}\right) \rightarrow H_{1}\left(X_{1}\right)$ and $j_{0}: H_{1}\left(X_{0} \cap X_{1}\right) \rightarrow H_{1}\left(X_{0}\right)$. An ordered basis for $H_{1}\left(X_{0} \cap X_{1}\right)$ is $\left\{\left[p_{0}\right], \cdots,\left[p_{n}\right],\left[q_{0}\right], \cdots\left[q_{n}\right],\left[m_{0}\right], \cdots,\left[m_{n}\right]\right\}$. An ordered basis for $H_{1}\left(X_{0}\right) \oplus H_{1}\left(X_{1}\right)$ is $\left\{j_{0}\left(\left[p_{0}\right]\right), j_{0}\left(\left[s_{0}\right]\right), \cdots j_{0}\left(\left[s_{n}\right]\right), j_{1}\left(\left[q_{0}\right]\right), j_{1}\left(\left[s_{0}\right]\right), \cdots j_{1}\left(\left[s_{n}\right]\right)\right\}$. The following lemma is proved with a standard analysis of the matrix of $j$.

Lemma 1. Let $[f],[g]$ be in the image of $\Phi: \mathbb{Z}^{n} \oplus P_{n} \rightarrow \Gamma_{n}$. Let $F=\left[F_{i j}\right]$ and $-G=-\left[G_{i j}\right]$ be the $n \times n$ linking matrices of $\hat{f}$ and -1 times the linking matrix of $\hat{g}$, respectively. Set $B$ equal to the submodule of $H_{1}\left(X_{0} \cap X_{1}\right)$ with ordered basis $\left\{\left[p_{1}\right]-\left[p_{0}\right], \cdots,\left[p_{n}\right]-\left[p_{0}\right],\left[q_{1}\right]-\left[q_{0}\right], \cdots,\left[q_{n}\right]-\left[q_{0}\right]\right\}$. Define $(F-G)$ : $B \rightarrow H_{1}\left(X_{0}\right) \oplus H_{1}\left(X_{1}\right)$ to be multiplication by the matrix formed by juxtaposing the linking matrices of $F$ and $-G$.

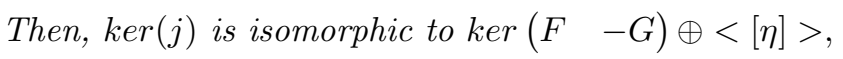

where $[\eta]=\left[m_{0}\right]-\left(\sum_{i=1}^{n}\left[m_{i}\right]\right)$.

\section{Example:}

Consider the example of representatives $(0,0,0) f$ and $(-1,0,0) g$ in the image of $\Phi: \mathbb{Z}^{3} \oplus P_{3} \rightarrow \Gamma_{3}$ corresponding to the pure framed braids in figure 1 , where the framings $a_{1}=a_{2}=a_{3}=b_{2}=b_{3}=0$ and $b_{1}=-1$. From $(0,0,0) f$ and $(-1,0,0) g$ we obtain the matrix

$$
\left(\begin{array}{ll}
F & -G
\end{array}\right)=\left(\begin{array}{cccccc}
0 & 1 & 0 & 1 & 0 & 0 \\
1 & 0 & 0 & 0 & 0 & -1 \\
0 & 0 & 0 & 0 & -1 & 0
\end{array}\right) .
$$

Using the symbol $T$ to stand for transpose, two elements in the kernel are

$$
\alpha=\left(\begin{array}{llllll}
1 & 0 & 0 & 0 & 0 & 1
\end{array}\right)^{T} \text { and } \beta=\left(\begin{array}{llllll}
0 & 1 & 0 & -1 & 0 & 0
\end{array}\right)^{T} .
$$

To construct the classes $\tau(\alpha)$ and $\tau(\beta)$ we decompose $W_{(0,0,0) f,(-1,0,0) g}^{4}$ into $X_{1}$ and $X_{0}$. In this case both are fiber bundles with base space $D_{2}$, and fiber $D_{3}$. For $i=1,2,3, j_{1}\left(\left[p_{i}\right]\right)$ is a longitude on a tubular neighborhood of the $i^{t h}$ strand of the closed braid $\hat{f}$ in the fiber over $\partial d_{1}$. Similarly, $j_{1}\left(\left[q_{i}\right]\right)$ corresponds to the $i^{\text {th }}$ strand of $\hat{g}$ in the fiber over $\partial d_{2}$. The classes $j_{0}\left(\left[p_{i}\right]\right)$ and $j_{0}\left(\left[q_{i}\right]\right)$ are longitudes on tubular neighborhoods of the $i^{\text {th }}$ strands of the closed identity braids in the fiber over $\partial d_{1}$ and $\partial d_{2}$, respectively, in $X_{0}$. With $y_{0}$ a point on the exterior boundary circle of $D_{3}$, 
the classes $j_{1}\left(\left[p_{0}\right]\right)$ and $j_{1}\left(\left[q_{0}\right]\right)$ represent $S^{1} \times\left\{y_{0}\right\}$ in the $S^{1} \times D_{3}$ fiber over $\partial d_{1}$ and $\partial d_{2}$ in $X_{1}$, respectively.

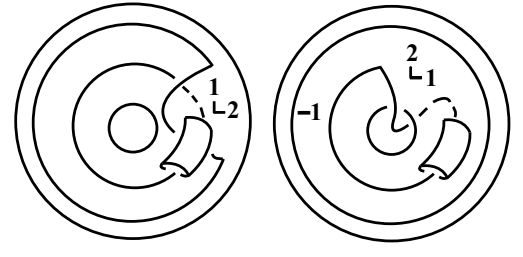

Figure 4: $\tau(\alpha)$ in $X_{1}$

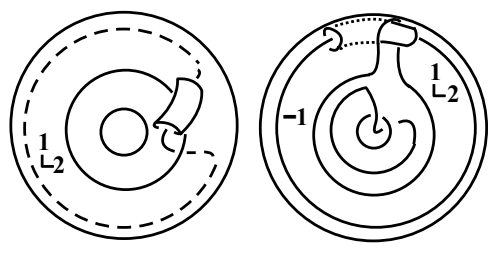

Figure 5: $\tau(\beta)$ in $X_{1}$

Since $\alpha=\left(\left[p_{1}\right]-\left[p_{0}\right]\right)+\left(\left[q_{3}\right]-\left[q_{0}\right]\right)$, attach two annular regions. One annular region has boundary $j_{1}\left(\left[p_{1}\right]\right)-j_{1}\left(\left[p_{0}\right]\right)$, and the second has boundary $j_{1}\left(\left[q_{3}\right]\right)-j_{1}\left(\left[q_{0}\right]\right)$. The first annular region is in the fiber over $\partial d_{1}$ in $X_{1}$, see the left side of figure 4 , corresponding to $\hat{f}$. To allow the tubular neighborhood with longitude $j_{1}\left(\left[p_{2}\right]\right)$ to pass through, we remove a disc and attach an $S^{1} \times[0,1]$ along the $S^{1} \times\{0\}$ boundary. The annular region with boundary $j_{1}\left(\left[q_{3}\right]\right)-j_{1}\left(\left[q_{0}\right]\right)$ is in the fiber over $\partial d_{2}$, corresponding to $\hat{g}$. We remove a disc and attach a tube so that a tubular neighborhood with longitude $j_{1}\left(\left[q_{2}\right]\right)$ may pass through, see the right side of figure 4. The tubes about $j_{1}\left(\left[p_{2}\right]\right)$ and $j_{1}\left(\left[q_{2}\right]\right)$ are then joined by passing through fibers over $D_{2}$.

In the fiber over $\partial d_{1}$, the annular region in $X_{1}$ is glued to the boundary of an annulus in $X_{0}$ between $j_{0}\left(\left[p_{1}\right]\right)$ and $j_{0}\left(\left[p_{0}\right]\right)$. In the fiber over $\partial d_{2}$, the annular region in $X_{1}$ is glued to an annulus in $X_{0}$ between $j_{0}\left(\left[q_{3}\right]\right)$ and $j_{0}\left(\left[q_{0}\right]\right)$. The surface $\tau(\alpha)$ is two tori joined by a tube, and with an orientation is a class of $H_{2}\left(W_{f, g}^{4}\right)$. The projection of this class to the base space $D_{2}$ is two circles, concentric to the interior boundary circles joined together with a simple non-intersecting arc, see figure 6 . The arc being the projection of the tube joining the annular regions in $X_{1}$.

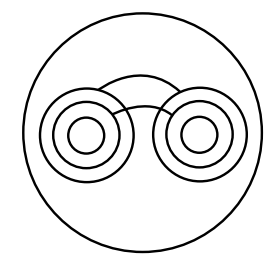

Figure 6: The projection of $\tau(\alpha)$ and $\tau(\beta)$ to $D_{2}$ in general position.

We construct a second surface $\tau(\beta)$ in a similar fashion. In the fiber over $\partial d_{1}$ in $X_{1}$, there is an annular region between $j_{1}\left(\left[p_{2}\right]\right)$ and $j_{1}\left(\left[p_{0}\right]\right)$, and a tube is created about a tubular neighborhood with $j_{1}\left(\left[p_{1}\right]\right)$. In the fiber over $\partial d_{2}$ there is an annular region with boundary $j_{1}\left(\left[q_{0}\right]\right)$ and a circle linking $j_{1}\left(\left[q_{1}\right]\right)$ with linking number -1 . 
This is due to the framing number -1 . For the tubular neighborhood with $j_{1}\left(\left[q_{1}\right]\right)$ to pass through the annular region, a disc is removed and a tube is attached, see figure 5. These tubes are joined together. After attaching the boundary to the corresponding annuli in $X_{0}$ we obtain a surface, denoted $\tau(\beta)$. The projection of $\tau(\beta)$ to $D_{2}$ is two circles joined by an arc. $\tau(\alpha)$ and $\tau(\beta)$ have an intersection of 1 . Without the tubes, the projections to the base space $D_{2}$, in general position, would be two pairs of concentric circles. Such surfaces would not intersect.

\section{The intersection form on $H_{2}\left(W_{f, g}^{4}\right)$}

Lemma 2. There is a splitting $\tau: \operatorname{ker}(j) \rightarrow H_{2}\left(W_{f g}^{4}\right)$.

\section{Proof}

As in section $4, \tau\left(\left[p_{i}\right]-\left[p_{0}\right]\right)$ is an annular region in $X_{1}$ over $\partial d_{1}$ with boundary $j_{1}\left(\left[p_{i}\right]\right)-j_{1}\left(\left[p_{0}\right]\right)$ glued to an annulus in $X_{0}$ with boundary $j_{0}\left(\left[p_{i}\right]\right)-j_{0}\left(\left[p_{0}\right]\right)$. Define $\tau\left(\left[q_{i}\right]-\left[q_{0}\right]\right)$ to be an annular region in $X_{1}$ over $\partial d_{2}$ with boundary $j_{1}\left(\left[q_{i}\right]\right)-j_{1}\left(\left[q_{0}\right]\right)$ glued to an annulus in $X_{0}$ with boundary $j_{0}\left(\left[q_{i}\right]\right)-j_{0}\left(\left[q_{0}\right]\right) . \tau([\eta])$ is defined to be the surface fiber over a point in $D_{2}$. To define $\tau$ on a general element of $k \operatorname{er}(j)$, extend linearly. In the construction of $\tau\left(a_{1}\left(\left[p_{1}\right]-\left[p_{0}\right]\right)+\cdots+a_{n}\left(\left[p_{n}-p_{0}\right]\right)+b_{1}\left(\left[q_{1}\right]-\left[q_{0}\right]\right)+\cdots+b_{n}\left(\left[q_{n}\right]-\left[q_{0}\right]\right)\right)$, for each $a_{k} \neq$ 0 , there are $\left|a_{k}\right|$ copies of annular regions between $j_{1}\left(\left[p_{k}\right]\right)$ and $j_{1}\left(\left[p_{0}\right]\right)$. The algebraic intersection of $j_{1}\left(\left[p_{i}\right]\right)$ with these regions is $a_{k} F_{i k}$. Similarly, for $b_{k} \neq 0$ there are $\left|b_{k}\right|$ copies of annular regions between $j_{1}\left(\left[q_{k}\right]\right)$ and $j_{1}\left(\left[q_{0}\right]\right)$, and the algebraic intersection with $j_{1}\left(\left[q_{i}\right]\right)$ is $b_{k} G_{i k}$. If $\left(a_{1}, \cdots, a_{n}, b_{1}, \cdots, b_{n}\right)$ is in $\operatorname{ker}(F-G)$, then for $1 \leqslant i \leqslant$ $n, \sum_{k=1}^{n} a_{k} F_{i k}=\sum_{k=1}^{n} b_{k} G_{i k}$. Therefore, we can glue $\left|\sum_{k=1}^{n} a_{k} F_{i k}\right|$ tubes about $j_{1}\left(\left[p_{i}\right]\right)$ to $\left|\sum_{k=1}^{n} b_{k} G_{i k}\right|$ tubes about $j_{1}\left(\left[q_{i}\right]\right)$. By examining the definition of $\delta$ one sees that $\tau$ is a splitting. Q.E.D.

Lemma 3. Let $\alpha, \beta \in H_{2}\left(W_{f, q}^{4}\right)$ and $\alpha=a_{1} \kappa_{1}+a_{2} \kappa_{2}$, where $a_{1}, a_{2} \in \mathbb{Z}, \kappa_{1} \in \operatorname{im}(\tau)$ and $\kappa_{2} \in \mathrm{im}(\sigma)$ of equation (1). If $a_{1}=0$, then the intersection $\alpha \cdot \beta=0$.

\section{Proof}

From equation (1) and lemma 2 we have $H_{2}\left(W_{f, g}^{4}\right) \approx i m(\sigma) \oplus \tau(k e r(j))$. A Mayer Vietoris sequence shows that a class in image of $\sigma$ can be divided into a class in the fiber over $\partial d_{1}$ of the base $D_{2}$ and a class in the fiber over $\partial d_{2}$. In general position, the projection of two such classes to $D_{2}$ is a finite set of circles concentric to $\partial d_{1}$ and to $\partial d_{2}$. Such classes do not intersect. A class in the image of $\tau$ projected to the base space $D_{2}$ is a finite set of circles concentric to $\partial d_{1}$ and $\partial d_{2}$, possibly with some simple arcs between them. In general position, one can arrange the class in the image of $\sigma$ so its projection to $D_{2}$ is a set of circles interior to the circles coming from the class in the image of $\tau$. Thus, there is no intersection. Q.E.D.

Remark 1. In general position, the fiber over a point does not intersect a surface. Thus, $\tau([\eta])$ does not contribute to signature computations.

Next we relate the algebraic computation of intersections in $W_{f, g}^{4}$ to our topo- 
logical description of section 4 . In the computation of $\tau(\alpha) \cdot \tau(\beta)$,

$$
\left(\begin{array}{cc}
F & 0_{3 \times 3} \\
0_{3 \times 3} & -G
\end{array}\right) \cdot \alpha=\left(\begin{array}{cccccc}
0 & 1 & 0 & 0 & -1 & 0
\end{array}\right)^{T}
$$

indicates the tubes created about $j_{1}\left(\left[p_{2}\right]\right)$ and $j_{1}\left(\left[q_{2}\right]\right)$ in the fiber over $\partial d_{1}$ and $\partial d_{2}$, respectively. The multiplication

$$
\begin{aligned}
& \beta^{T} \cdot\left(\left(\begin{array}{cc}
F & 0_{3 \times 3} \\
0_{3 \times 3} & -G
\end{array}\right) \cdot \alpha\right)= \\
& \left(\begin{array}{llllll}
0 & 1 & 0 & -1 & 0 & 0
\end{array}\right) \cdot\left(\left(\begin{array}{llllll}
0 & 1 & 0 & 0 & -1 & 0
\end{array}\right)^{T}\right)=1
\end{aligned}
$$

reflects that the tube about $j_{1}\left(\left[p_{2}\right]\right)$ intersects the annular region in the fiber over $\partial d_{1}$ with boundary components $j_{1}\left(\left[p_{0}\right]\right)$ and $j_{1}\left(\left[p_{2}\right]\right)$. More generally, we have the next theorem. Recall the map $\delta: H_{2}\left(W^{4}\right) \rightarrow H_{1}\left(X_{1} \cap X_{2}\right)$ from equation (1).

Theorem 2. Let $[f],[g]$ be in the image of $\Phi: \mathbb{Z}^{n} \oplus P_{n} \rightarrow \Gamma_{n}$, and $\alpha, \beta \in$ $H_{2}\left(W_{f, g}^{4}\right)$. Set $F=\left[F_{i j}\right]$ to be the linking matrix of $\hat{f}$, and $-G=-\left[G_{i j}\right]$ to be -1 times the linking matrix for $\hat{g}$. Suppose the projection of $\delta(\alpha)$ to $\operatorname{ker}(F-G)$ has coordinates $\left(a_{1}, \ldots, a_{n}, b_{1}, \ldots, b_{n}\right)$, and the projection of $\delta(\beta)$ has coordinates $\left(c_{1}, \ldots, c_{n}, d_{1}, \ldots, d_{n}\right)$. Then, the intersection $(\alpha) \cdot(\beta)=$

$$
\left(\begin{array}{llllll}
c_{1} & \ldots & c_{n} & d_{1} & \ldots & d_{n}
\end{array}\right)\left(\begin{array}{cc}
F & 0_{n \times n} \\
0_{n \times n} & -G
\end{array}\right)\left(\begin{array}{llllll}
a_{1} & \ldots & a_{n} & b_{1} & \ldots & b_{n}
\end{array}\right)^{T}
$$

Proof

By lemma 3 and remark 1 , the only non-zero intersections are from classes in the image of $\tau$ restricted to $\operatorname{ker}(F-G)$. Consider the sum $c_{k}\left(a_{k} F_{1 k}+\ldots+a_{k} F_{n k}\right)$. When $c_{k} \neq 0$ we have $\left|c_{k}\right|$ annular regions between with boundary components $j_{1}\left(\left[p_{k}\right]\right)$ and $j_{1}\left(\left[p_{0}\right]\right)$. If $a_{k} \neq 0$, there will be $\left|a_{k} F_{i k}\right|$ number of tubes around a tubular neighborhood of $j_{1}\left(\left[p_{k}\right]\right)$, formed by avoiding intersections with annular regions between $j_{1}\left(\left[p_{i}\right]\right)$ and $j_{1}\left(\left[p_{0}\right]\right)$. Summing for $i=1 \ldots n$ yields an algebraic intersection of $c_{k}\left(a_{k} F_{1 k}+a_{k} F_{2 k}+a_{k} F_{n k}\right)$. Accounting for all $j_{1}\left(\left[p_{k}\right]\right), 1 \leqslant k \leqslant n$, we obtain

$$
\left(c_{1}, \quad \ldots \quad, c_{n}\right)(F)\left(\begin{array}{lll}
a_{1} & \ldots & a_{n}
\end{array}\right)^{T} .
$$

A similar analysis yields the intersection number coming from $j_{1}\left(\left[q_{k}\right]\right)$, $1 \leqslant k \leqslant n$, is

$$
-\left(d_{1}, \quad \ldots \quad, d_{n}\right)(G)\left(\begin{array}{lll}
b_{1} & \ldots & b_{n}
\end{array}\right)^{T} \text {. Q.E.D. }
$$

Using the map $\Phi: \mathbb{Z}^{n} \oplus P_{n} \rightarrow \Gamma_{n}$ we obtain the commutative diagram below.

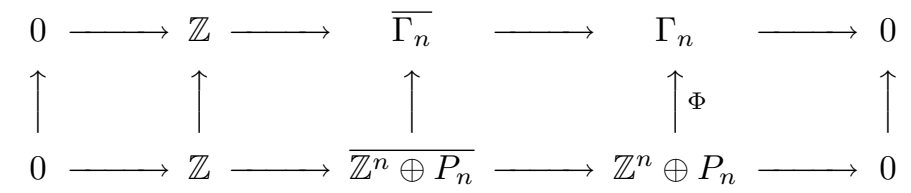

The group $\overline{\Gamma_{n}}$ is a central extension of the mapping class group $\Gamma_{n}$ studied in [1], and $\overline{\mathbb{Z}^{n} \oplus P_{n}}$ is the pullback of $\Phi$. An element of the subgroup $\overline{\mathbb{Z}^{n} \oplus P_{n}}$ is a pair $(h, a)$, 
where $a$ is an integer and $h$ is in the image of $\Phi$. The multiplication in $\overline{\mathbb{Z}^{n} \oplus P_{n}}$ is computed using linking matrices.

In conclusion, we multiply $([(0,0,0) f], a) \cdot([(-1,0,0) g], b)$ in $\overline{\mathbb{Z}^{3} \oplus P_{3}}$. The intersection pairing on $W_{f g}^{4}$ is given by the quadratic form $x^{T} A x$ where

$$
A=\left(\begin{array}{cc}
F & 0_{3 \times 3} \\
0_{3 \times 3} & -G
\end{array}\right)=\left(\begin{array}{cccccc}
0 & 1 & 0 & 0 & 0 & 0 \\
1 & 0 & 0 & 0 & 0 & 0 \\
0 & 0 & 0 & 0 & 0 & 0 \\
0 & 0 & 0 & 1 & 0 & 0 \\
0 & 0 & 0 & 0 & 0 & -1 \\
0 & 0 & 0 & 0 & -1 & 0
\end{array}\right)
$$

The signature of the matrix $A$ is 1 , and therefore

$([(0,0,0) f], a) \cdot([(-1,0,0) g], b)=\left([(-1,0,0) f g], a+b+3 \operatorname{Sign}\left(W_{f g}^{4}\right)\right)$

$=([(-1,0,0) f g], a+b+3)$.

\section{References}

[1] Atiyah, M.F. On Framings of 3-Manifolds, Topology, Vol 29, no.1, pp. 1-7, 1990

[2] Birman, J. Braids, Links and Mapping Class Groups, Ann. Math. Stud. 82, 1975

[3] Birman, J., Powell, J. Special Representations for 3-Manifolds, Geometric Topology. Proc. Academic Press, pp. 23-51, 1977

[4] Mayer, W. Die Sigaur von Flachenbundeln, Math. Ann. Vol. 201, pp. 239-264, 1973

[5] Natov, J. Pure Framed Braids and 3-Manifolds, Doctoral Dissertation, Louisiana State Univ. Baton Rouge, LA, 1997

[6] Prasolov, V. V. and Sossinsky, A. B. Knots, Links, Braids and 3-Manifolds: an introduction to new invariants in low dimensional topology, Trans. Math. Monogr., Vol. 154, Amer. Math. Soc., 1996

This article may be accessed via WWW at http://www.rmi.acnet.ge/hha/ or by anonymous ftp at

ftp://ftp.rmi.acnet.ge/pub/hha/volumes/2003/n1a11/v5n1a11.(dvi,ps,pdf)

Jonathan Natov natovj@mail.com

Department of Mathematics

New York City College of Technology, C.U.N.Y.

300 Jay Street

Brooklyn, New York 11201 